\title{
Novel insights into the role of Clostridium novyi-NT related combination bacteriolytic therapy in solid tumors (Review)
}

\author{
XU FENG, PAN HE, CHEN ZENG, YE-HAN LI, SUSHANT K. DAS, BING LI, HAN-FENG YANG and YONG DU \\ Department of Radiology, Affiliated Hospital of North Sichuan Medical College, Nanchong, Sichuan 637000, P.R. China
}

Received May 10, 2020; Accepted November 20, 2020

DOI: $10.3892 / \mathrm{ol} .2020 .12371$

\begin{abstract}
Several solid tumors (for example leiomyosarcoma, melanoma and hepatocellular carcinoma) possess areas of hypoxia, which underlies one of the primary reasons of failure of conventional anticancer therapies. The areas of poor vascularization are insensitive to radiotherapy and chemotherapeutic drugs. Conversely, the hypoxic regions of tumors provide an ideal environment for anaerobic bacteria. The attenuated anaerobic bacterium, Clostridium novyi-NT (C. novyi-NT), is highly sensitive to oxygen and can target the destruction of hypoxic and necrotic areas of tumors, inducing oncolysis and characteristics indicative of an immune response. Theoretically, chemotherapy, radiotherapy and immunotherapy combined with bacterial therapy can be used as a novel means of treating solid tumors, promoting tumor regression and inhibiting metastasis formation with a notable beneficial effect. The present review discusses the molecular mechanisms of combined bacteriolytic therapy, predominantly focusing on C. novyi-NT, and summarizes the findings of previous studies on experimental animal models, including its efficacy and safety via different drug delivery routes. This strategy has great potential to overcome the limitations of conventional cancer therapy, resulting in improved treatments, and thus potentially improved outcomes for patients.
\end{abstract}

\section{Contents}

1. Introduction

2. Hypoxia: The characteristic marker of malignant solid tumors

3. Mechanism and issues of C. novyi-NT as an antitumor agent

4. C. novyi-NT combined with chemotherapy

Correspondence to: Professor Yong Du, Department of Radiology, Affiliated Hospital of North Sichuan Medical College, 63 Wenhua Road, Nanchong, Sichuan 637000, P.R. China

E-mail: duyong@nsmc.edu.cn

Key words: C. novyi-NT, combination bacteriolytic therapy, intratumoral injection, intravenously injected, solid tumor regression
5. C. novyi-NT combined with trans arterial chemoembolization (TACE)

6. C. novyi-NT combined with radiotherapy

7. C. novyi-NT and immunotherapy

8. C. oncolyticum M55 combined with radiofrequency ablation

9. Comparison of intratumoral injection and intravenous management

10. Future perspectives and conclusions

\section{Introduction}

Cancer is one of the leading causes of mortality, and $~ 90 \%$ of cancer-associated mortalities are caused by solid tumors (1). Early diagnosis and established treatment options, such as surgery, radiotherapy and chemotherapy represent the backbone of cancer therapy (2). Despite advancements in these therapies, they bear several disadvantages and risks. For example, not every tumor can be surgically removed. In addition, the efficacy of chemotherapy and radiotherapy is limited due to their general mode of activity, which in turn results in treatment resistance (3). Chemotherapy is plagued by decreased drug penetration into tumor cells (for example colorectal cancer, leiomyosarcoma and melanoma cells) adjacent to the poorly vascularized regions, peripheral toxicity and decreased immunity of patients, which leads to a poor clinical prognosis following treatment (4).

Necrotic areas are present in tumor tissues and absent in normal tissues (5). Live bacteria, including Clostridium novyi-NT (C. novyi-NT) can overcome the limitations of hypoxia in solid tumors and selectively grow in areas of absolute hypoxia and necrosis, suggesting that it may serve as a powerful tool for targeted therapy of tumors $(6,7)$. The advantages of C. novyi-NT anticancer therapy are largely due to its generally favorable toxic characteristics, strong oncolytic effects, minimal tumor resistance and strong targeting (8), which can be used as a gene therapy carrier for targeted gene therapy (9). In addition, bacterial therapy activates the host immune system antitumor effects (6). Traditional chemotherapy and radiotherapy can target and remove the oxygen-rich tumor cells at the edge; however, complete tumor removal remains a challenge. Conversely, the combination of traditional therapy with bacteriolytic therapy can simultaneously kill both well-oxygenated and hypoxic regions of 
the tumor, and even eradicate otherwise untreatable tumors to achieve no evidence of disease (10). This combination of traditional chemotherapy or radiotherapy with bacteriolytic therapy is referred to as combination bacteriolytic therapy (COBALT) (10). The present review discusses the molecular mechanisms of C. novyi-NT related COBALT, its advantages in tumor therapy and pre-clinical results, particularly the advantages and disadvantages of routes of drug delivery, such as intratumoral or intravenous injection.

\section{Hypoxia: The characteristic marker of malignant solid tumors}

It is well-known that solid tumors grow in a unique acidic microenvironment, triggering cellular release of tumor angiogenic factors, which can induce pathological angiogenesis leading to tumor hypoxia, acidity, nutrient deficiency and cell death (11). The presence of tumor hypoxia can alter genomic stability (accelerating the genetic diversity acquired) and promote the emergence of a malignant phenotype (12); induction of expression of tumor angiogenesis genes and activation of the expression of invasion and metastasis related gene expression, increasing the potential of tumor metastasis (13). This results in poor selectivity and drug resistance, and decreases the efficacy of radiotherapy and chemotherapy (14-16). Thus, tumor hypoxia, which is a characteristic hallmark of malignant solid tumors, remains an obstacle of several current treatment regimens, such as chemotherapy, radiotherapy, photodynamic therapy and immunotherapy (1).

\section{Mechanism and issues of C. novyi-NT as an antitumor agent}

Over 100 years ago, William Coley first reported the phenomenon of tumor regression in a patient with unresectable tumors after accidental infection with Streptococcus pyogenes (17). This discovery opened the field of investigating bacterial cancer therapy. Clostridium is a type of Gram-positive anaerobic bacteria that was first used for antitumor therapy (5). Preclinical animal studies have demonstrated, being anaerobic in nature, these bacteria specifically migrate and germinate in the hypoxic/necrotic areas of solid tumors with targeted germination and tumor regression $(5,18)$. C. novyi (ATCC19402) is unique compared to other candidate therapeutic bacteria, and is one of the most favorable bacterium strains, whose spore infiltration in tumors is superior to that of bifidobacteria. However, like other bacteria, the spores of clostridium strains have also been demonstrated to exert excessive toxicity. To address this issue, the primary toxin of clostridium bacteria (toxin-A), which causes toxicity, was eliminated to produce a non-toxic spore (10). This non-toxic strain of C. novyi has been designated as C. novyi-NT (10). C. novyi-NT spores are easier to store, handle and are more stable under nonpermissive conditions (19). Currently, C. novyi-NT is the only strain of Clostridium to be tested in humans as an anticancer drug (19-21).

The molecular mechanisms underlying bacterial tumor destruction are not yet fully understood, and may include direct tumor cell destruction, as well as activation of the host immune system $(22,23)$. Bacteria act as tumoricidal agents and inhibit cancer cell proliferation through cytotoxins. High concentrations of cytotoxins directly kill tumor cells, whereas low concentrations of cytotoxins alter cellular processes, including cell proliferation, apoptosis and differentiation (22). Bacteria can also be genetically modified to express proteins that are toxic to cancer cells, including the secretion of lipases, proteases and other degradative enzymes (23). In addition, bacteria stimulate a potent cellular immune response that subsequently destroys residual tumor cells not lysed by the bacteria (23). C. novyi-NT expresses several extracellular proteins, such as phospholipase C (NT01CX0979) and two lipases (NT01CX2047 and NT01CX0630), all of which alter the structure of lipid bilayers and membrane permeability, thereby directly affecting the cytotoxicity on tumors (24). Furthermore, phospholipases may also activate inflammatory responses and induce antitumor immunity (24). In addition to the direct cytotoxicity, C. novyi-NT also induces a strong inflammatory response involving pro-inflammatory cytokines, such as interleukin-6, granulocyte-colony stimulating factor, macrophage inflammatory protein 2 and tissue inhibitor matrix metalloproteinase 1 that attract a massive influx of inflammatory cells, which in turn results in recruitment of a substantial number of immune cells to generate a durable adaptive antitumor immune response $(23,25)$. The inflammatory reaction restrains the spread of the bacterial infection, providing a second layer of control in addition to that provided by the requisite anaerobic environment (23). The inflammation may also directly contribute to the destruction of tumor cells through the production of reactive oxygen species, proteases and other degradative enzymes (23). Dying tumor cells, along with the release of damage-associated molecular pattern molecules released by dendritic cells, trigger tumor associated antigen specific $\mathrm{T}$ cells, which in turn generates immunogenic cell death, and is hypothesized to serve an important role in long lasting adaptive immunity (26-28). Previous studies have reported that $\mathrm{C}$. novyi-NT spores can germinate locally within the tumor, and precisely spread throughout the tumor and its microsatellites, causing hemorrhagic necrosis, strong inflammatory responses, tumor cell lysis and regression $(10,19,23,25)$.

Furthermore, CD8+ T lymphocytes isolated from C. novyi-NT treated mice have been demonstrated to stimulate acquired immunity in tumor-specific models (29). Previous studies have supported the notion that bacteriolytic treatment can stimulate a long-lasting antitumor immune response $(23,30)$. In a previous study, cured animals rejected a subsequent challenge from the same tumor (23). Thus, innate or acquired immunity serves a key role in achieving durable complete responses (CRs) and suppressing distant metastasis (29). Further investigations are required to determine the molecular mechanisms of tumor destruction associated with C. novyi-NT, and the effects of host-mediated immunity on distant metastasis.

Although C. novyi-NT has exhibited antitumor properties in experimental animal models $(23,25,31)$, concern regarding the efficacy of $\mathrm{C}$. novyi-NT in tumors lacking necrotic areas remains. Being an anaerobic bacterium, C. novyi-NT does not target the oxygen-rich regions of tumors. Generally, small tumors and oxygen-rich tumors lack necrotic areas, resulting in the inability of these spores to colonize small metastases and viable oxygen-requiring regions of tumors, leading to poor 
clinical outcomes (32). This issue has led researchers to study the use of combination therapy to improve patient outcomes. Further studies applying combination therapy in small solid tumors lacking hypoxic areas are required to provide future direction.

\section{C. novyi-NT combined with chemotherapy}

Although C. novyi-NT spores are considered an effective antitumor agent that promote regression of certain types of cancer (including leiomyosarcoma, melanoma and hepatocellular carcinoma) (19,31,33), injecting C. novyi-NT alone into mice and rabbits bearing transplanted syngeneic tumors has demonstrated low cure rates (25-30\%) (23). In addition, CR was not observed in spontaneously occurring canine tumors (34). Given that cancer is a complex disease, the strategy of using a combination of internal and external attack methods to kill both hypoxic and oxygen-rich cells is considered a preferable and potentially favorable option (10). Alternatively, the presence of bacteria within the tumor environment can also alter the chemical structure of some chemotherapeutic agents, affecting their activity and local concentration, thus sensitizing cancer therapy (35). C. novyi-NT has been combined with several chemotherapeutic drugs to produce synergistic effects (36). Bacterial therapy is combined with conventional chemotherapeutic drugs, such as chemotherapeutic drugs, anti-angiogenic drugs, heavy metals and heat shock proteins to treat cancer in combination with bacteriolytic therapy (37). Notably, extensive hemorrhagic necrosis of tumors often occurs within $24 \mathrm{~h}$ when $\mathrm{C}$. novyi-NT spores are administered together with conventional chemotherapeutic agents (Dolastatin-10, Cyclophosphamide, mitomycin $\mathrm{C}$ and vincristine), exhibiting notable and long-term antitumor effects (10). The combined treatment of C. novyi-NT spores with microtubule binding agents (D10 and combretastatin a-4 prodrug) in nude mice with a xenograft colorectal cancer demonstrated that D10 significantly enhances the ability of C. novyi-NT spores to lyse tumors, while addition of the tumor cell agent, CTX further enhances the efficacy of COBALT (38). C. novyi-NT spores combined with microtubule stabilizers, such as discodermolide analogues cause rapid and complete tumor regression in HCT116 tumor-bearing mice (39). In addition, microtubule stabilizers have been demonstrated to promote the germination of C. novyi-NT spores in the hypoxic region by expanding the area, resulting in rapid tumor regression (40). COBALT may genetically manipulate $\mathrm{C}$. novyi-NT to enhance its potency or selectivity (10). The aforementioned results suggest that even a single dose of COBALT can cause partial or complete regression of cancer in certain xenograft models $(33,40)$. Another notable strategy is the use of C. novyi-NT-mediated cleavage of the erythrocyte membrane, and increase in intratumoral release of liposome-encapsulated drugs, thereby raising local drug concentrations and decreasing systemic toxicity (41). C. novyi-NT combined with a single dose of liposomal doxorubicin has been reported to completely eradicate tumors in tumor-bearing mice (41), as well as significantly increase the tumor clearance rate of implanted glioblastomas in rats (25).

This significant antitumor effect of COBALT is associated with its notable toxicity, which is positively associated with tumor size, possibly due to tumor lysis syndrome; when a large tumor burden is rapidly destroyed by antineoplastic agents (10). However, COBALT still faces the problem of resistance to chemotherapeutic agents, and different types of tumors may require combination with different chemotherapeutic agents to exert their maximal effects. Another concerning issue is the size of the tumor, as it needs to be large enough to possess necrotic areas (32). COBALT is not suitable for primary and metastatic tumors without areas of necrosis and hypoxia (10). In addition, not all tumors are equally sensitive to COBALT (33), such as mice bearing human colorectal cancer xenografts (HCT116) and human biliary cancer HuCC-T1, and further clarification of the types of tumors where COBALT may be suitable is required. Additionally, understanding the molecular mechanism by which C. novyi-NT kills tumor cells may also provide novel insights and improvements into the management of tumors.

\section{C. novyi-NT combined with trans arterial chemoembolization (TACE)}

TACE has become the preferred treatment for advanced unresectable hepatocellular carcinoma, including large and/or multinodular tumors without vascular invasion or distant metastasis. Due to the ability to achieve a high local concentration of the chemotherapeutic drugs and precise blocking of the feeding artery of the tumor, large areas of tumor necrosis will occur. However, TACE can increase the drug concentration in the tumor. The drug concentration is higher than that of systemic chemotherapy, with less toxicity, when using TACE (42). Thus, C. novyi-NT combined with TACE for tumor treatment decreases the tumor mass by chemoembolization, killing the tumor in the oxygen-rich areas, and expanding the hypoxic necrotic area in the tumor, which serves as the perfect environment for $\mathrm{C}$. novyi-NT germination and reproduction (43). A study treating 12 rabbits (VX2 liver cancer models) with a combination of C. novyi-NT and TACE therapy confirmed that TACE increased the colonization of C. novyi-NT spores within tumors in a short period of time (43). C. novyi-NT spores combined with TACE in the treatment of rabbit VX2 liver cancer had a more significant antitumor effect compared with TACE alone, with significantly decreased tumor size, a significantly expanded extent of necrosis and effectively prolonged survival time in tumor-bearing rabbits (43). This strategy provides a novel treatment option for large tumors which are unresectable.

\section{C. novyi-NT combined with radiotherapy}

Cancer treatment requires a multiple disciplinary team model. Given that oxygen is a required effector in radiation-induced cell death (14), the areas of poor tumor vascularization increase the tumor's resistance to radiation therapy (44). However, C. novyi-NT is a radiation enhancer that can directly target the hypoxic regions of tumors, and thus enhances the effects of radiation (33). In addition, when used in combination with radiotherapy, radiation can lead to microvascular endothelial cell injury producing more hypoxic areas, favoring C. novyi-NT growth (45). Thus, animal experiments using C. novyi-NT combined with external beam irradiation, brachytherapy and radioimmunoassay can produce synergistic responses, attenuate tumor immune escape mechanisms and 
enhance the effect of radiation, thus decreasing radiation doses and leading to partial or complete regression in certain cancer xenograft models (33). C. novyi-NT disrupts the tumor hypoxic region, and in combination with radiotherapy or $I^{131}$-monoclonal antibody radioimmunotherapy, overcomes the hypoxic barrier, resulting in long-term remission of the growth of transplanted tumors in mice $(33,46,47)$. In addition, given that branched gold nanoparticles (BGNP) can be used as effective radiosensitizers and functional carriers for intratumoral delivery of cytotoxic drugs (21), the use of electrostatic deposition to achieve BGNP-coated C novyi-NT spores in CT-guided combined radiotherapy and chemotherapy can enhance the antitumor efficacy and accurately locate the intratumoral distribution of C novyi-NT spores. Notably, C. novyi-NT spores in combination with radiotherapy only increase toxicity by a small amount, which is controlled by administration of antibiotics. The efficacy of combined C. novyi-NT spores and radiotherapy is not affected by tumor size and host immune status $(25,48)$. Conversely, low-dose radiotherapy combined with the use of specific bacterial species attenuates tumor immune escape mechanisms (37). In conclusion, the adoption of this novel strategy can break through the bottleneck of current conventional treatments of refractory tumors.

\section{C. novyi-NT and immunotherapy}

Bacterial immunotherapy uses in vitro injection of engineered bacteria or bacteria as the carrier, whereby bacteria induce inflammation to cause a potent adaptive immune response. Engineered bacteria secrete compounds that stimulate the immune system to cause specific immune responses, and express tumor-associated antigens, specifically and in a targeted manner to eliminate specific solid tumors, whilst preserving normal tissues. C. novyi-NT is one of the most common bacterial species used as an immunotherapeutic agent (49). As intratumoral hypoxia triggers hypoxia-inducible factor $1 \alpha$ (HIF-1a) (50), a key transcription factor responsible for cell survival, it is an important target for bacterial immunotherapy (50). Groot et al (51) first demonstrated the allogenic expression of a C. novyi-NT strain of VHH antibody targeting HIF-1a. Clostridium-directed antibody therapy was combined with the oncolytic properties of Clostridium and the target selectivity of antibody therapeutics to target the most resistant regions in solid tumors. Notably, when the induced immune response is combined with the bacteriolytic effects of C. novyi-NT, it can eradicate established large tumors, with both primary tumors and metastases responding to the treatment (23). A previous study demonstrated that binding to C. novyi-NT spores by blocking immune checkpoints reverses tumor-induced immunosuppression and initiates synergistic antitumor immune effects (23). C. novyi-NT combined with immunotherapy has exhibited significant potential to improve the curative effect of refractory primary tumors and metastatic tumors. However, bacterial immunotherapy must overcome obstacles such as immune tolerance, immunosuppression of the tumor microenvironment and severe autoimmune reactions to non-targeted tissues. Nonetheless, repeated administration of Clostridium has not been demonstrated to induce host immune responses against Clostridium $(22,32,49)$. In a previous study, even 10-fold increase in Clostridium-species antibodies in the serum following a second bacterial administration failed to exhibit rejection of colonization of tumors, suggesting that long-term production of the therapeutic proteins from the engineered Clostridium is possible in tumors (32).

\section{C. oncolyticum M55 combined with radiofrequency ablation}

Radiofrequency ablation uses high-frequency radiofrequency waves to make ions in the tissue vibrate and rub against each other at high speed, increasing the temperature of the tumor area, such that the cancer cells dehydrate resulting in coagulative necrosis and an increase in the tumor necrosis area (52). This provides an ideal environment for the budding and reproduction of C. oncolyticum M55 spores. Radiofrequency pretreatment is initially performed, followed by intravenous administration of Somatomedes oncolyticum M55 spores to treat cervical induced carcinomas in 861 mice. Clinical trials have demonstrated that radiofrequency ablation significantly enhances the oncolytic effects of oncolyticum M55 (53). For rapidly growing Ehrlich adenocarcinoma, systemic application of bacterial spores has the most prominent effect $12 \mathrm{~h}$ after initial RF treatment (54). For slow-growing melanoma, radiofrequency therapy, intravenous injection of C. oncolyticum M55 spores and radiotherapy were combined, with a cure rate of 20\%; although the survival time was prolonged, in situ recurrence still resulted in the death of the remaining mice (55). Prospective studies should focus on assessing more effective cancer treatments, such as anaerobic bacteria combined with radiofrequency ablation, cryoablation, microwave ablation and irreversible electroporation for different pathological types of tumors.

\section{Comparison of intratumoral injection and intravenous management}

Currently, the primary methods of administration are systemic intravenous injection and direct intratumoral injection $(25,34)$. Different routes of administration significantly affect bacterial tumor colonization and toxicity (Fig. 1). Lee et al (56) suggested that systemic intravenous injection of tumor-targeted bacteria is the predominant route of administration. Another view is that direct intratumoral injection may allow relatively small doses of spores to be used, ensuring that a larger effective dose reaches the target tumor and is distributed around the tip of the needle (19). Conversely, systemic injection requires large doses of injected spores; however, the proportion of spores actually delivered to the tumor is small (lower effective dose) (48), particularly in humans. In another study, although both intravenous and intratumoral administration of C. novyi-NT spores resulted in a sustained increase in NK cell-like function, the duration of NK cell-like function by intravenous injection was longer (30). However, Thiele et al (57) and Malmgren et al (5) have demonstrated both to be of similar effect. Another concern is systemic toxicity, although previous studies have demonstrated that bacterial spores are not toxic to normal animals, their toxicity is primarily dependent on tumor volume, followed by germination, spore dose and route of administration, which is more pronounced 
A

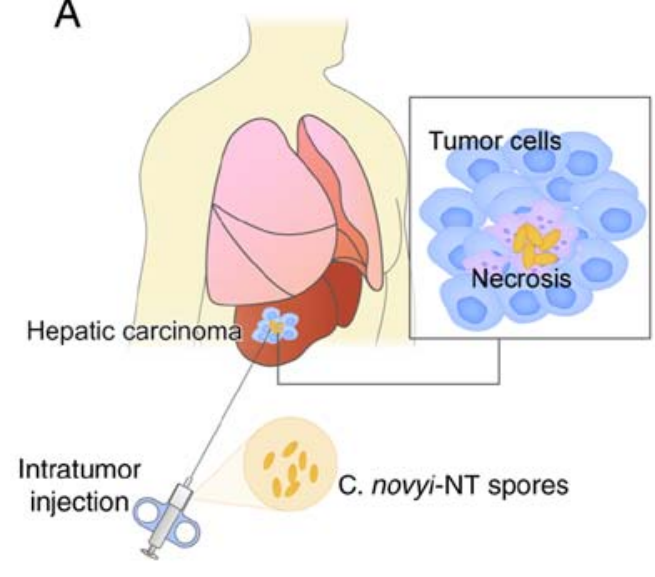

B

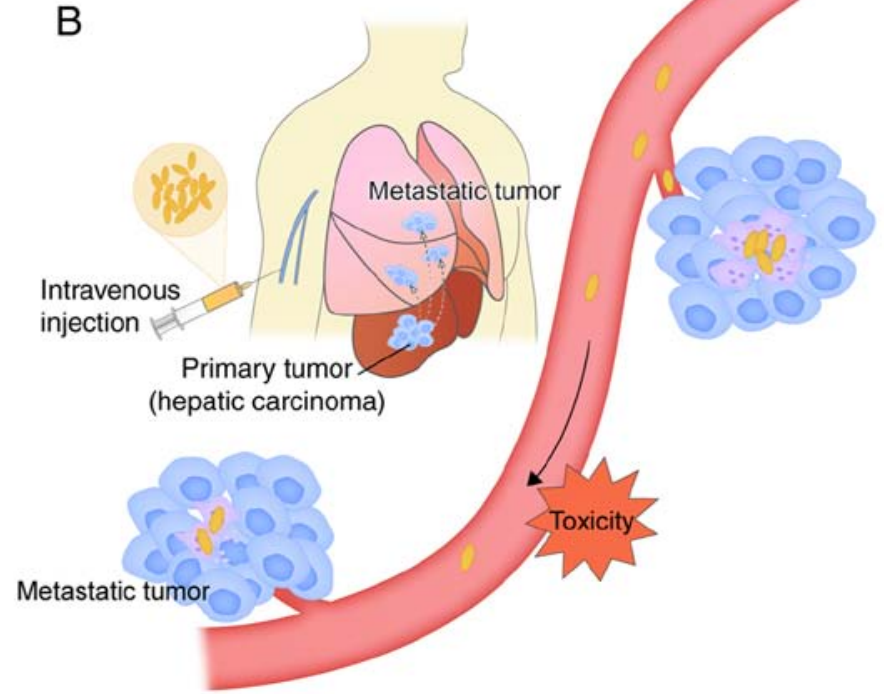

Figure 1. Comparison of intratumoral injection and intravenous management. (A) Direct intratumoral injection may allow relatively small doses of spores to be used, ensuring that a larger effective dose reaches the target tumor and is distributed around the tip of the needle. (B) Systemic injection requires large doses of injected spores; however, the proportion of spores delivered to the tumor is small (lower effective dose). Only the route of systemic administration can be selected for the treatment of disseminated cancers. C. novyi-NT, Clostridium novyi-NT.

in animals with larger tumors $(22,48)$, and the possibility that intratumorally germinated bacteria release potent toxins cannot be ruled out. Krick et al (34) hypothesized that there is potential for toxicity from the injection of a large numbers of spores into systemic blood pools. However, Roberts et al (19) demonstrated that the most common toxicity observed with intratumoral injection of C. novyi-NT spores was the expected symptoms associated with bacterial infection. Theoretically, only the route of systemic administration can be selected for the treatment of disseminated cancers. Based on these theories, the appropriate route of administration should be selected following specific evaluation according to the selected strains and types of tumors.

C. novyi-NT can be used to assess bacteriolytic therapies, including development of non-invasive magnetic resonance imaging. C. novyi-NT spores are internally labeled with iron oxide nanoparticles and monitored with magnetic resonance, and methods such as BGNP-coated C. novyi-NT spores for CT-Guided intratumoral injection can be used for imaging to monitor the distribution, accumulation, germination and clearance of tumor-homing bacteria (31,58-60).

\section{Future perspectives and conclusions}

Although COBALT seems promising, further research is required to determine the toxic side effects and underlying molecular mechanisms of tumor destruction, in order to better understand the issue of increased systemic toxicity. Experimental studies are warranted to develop strategies to keep rate of tumor regression under control, so as to avoid the occurrence of tumor lysis syndrome. Moreover, experimental studies also need to explore the strategies to avoids the possibility of DNA mutations during treatment. DNA mutation during treatment can cause excessive infection and treatment failure. Furthermore, different administration routes can also affect antitumor efficacy, and suitable administration routes for different tumors should be assessed and validated. Further studies are also required to assess the association between tumor size and the dosage of C. novyi-NT spores used, in order to determine the maximum tolerated dose to further improve the therapeutic index and safety. Regarding intratumoral residual safety concern for C. novyi-NT spores, metronidazole has been reported to completely eliminate C. novyi-NT, and thus should be assessed as a potential countermeasure (32).

In conclusion, the hypoxic region of solid tumors can be treated as therapeutic targets, and C. novyi-NT is a reliable and effective tool for tumor targeting and delivery of therapeutic genes/drugs. In addition to destroying the hypoxic area to effectively treat the remaining oxygen-rich cancer cells around the tumor, a combination scheme should be adopted. Currently, it has been demonstrated that C. novyi-NT spores in combination with lysogenic therapy may be beneficial in improving the antitumor efficacy, and have achieved curative results in some experimental animals and a small number of patients. The treatment of tumor hypoxemia will likely compensate for the current obstacles of conventional treatment of tumors, and C. novyi-NT spores based COBALT is a promising alternative for the treatment of solid tumors.

\section{Acknowledgements}

Not applicable.

\section{Funding}

No funding was received.

\section{Availability of data and materials}

Not applicable. 


\section{Authors' contributions}

$\mathrm{XF}$ and YD wrote and edited the manuscript. PH, CZ, YHL, SKD, BL and HFY contributed toward the conception and design of the study and critically revised the manuscript. All authors read and approved the final manuscript.

\section{Ethics approval and consent to participate}

Not applicable.

\section{Patient consent for publication}

Not applicable.

\section{Competing interests}

The authors declare that they have no competing interests.

\section{References}

1. Brown JM and William WR: Exploiting tumour hypoxia in cancer treatment. Nat Rev Cancer 4: 437-447, 2004.

2. Allen MR: Medication-Related osteonecrosis of the jaw: Basic and translational science updates. Oral Maxillofac Surg Clin North Am 27: 497-508, 2015.

3. Bertout JA, Patel SA and Simon MC: Hypoxia and metabolism series-timeline the impact of O-2 availability on human cancer Nat Rev Cancer 8: 967-975, 2008.

4. Brown JM and Giaccia AJ: The unique physiology of solid tumors: Opportunities (and problems) for cancer therapy. Cancer Res 58: 1408-1416, 1998.

5. Malmgren RA and Flanigan CC: Localization of the vegetative form of clostridium tetani in mouse tumors following intravenous spore administration. Cancer Res 15: 473-478, 1955.

6. Zu C and Wang JS: Tumor-colonizing bacteria: A potential tumor targeting therapy. Crit Rev Microbiol 40: 225-235, 2014.

7. Ben-Jacob E, Coffey DS and Levine H: Bacterial survival strategies suggest rethinking cancer cooperativity. Trends Microbiol 20: 403-410, 2012.

8. Forbes NS: Profile of a bacterial tumor killer. Nat Biotechnol 24 : 1484-1485, 2006.

9. Pawelek JM, Low KB and Bermudes D: Bacteria as tumour-targeting vectors. Lancet Oncol 4: 548-556, 2003.

10. Dang LH, Bettegowda C, Huso DL, Kinzler KW and Vogelstein B: Combination bacteriolytic therapy for the treatment of experimental tumors. Proc Natl Acad Sci USA 98: 15155-15160, 2001.

11. Danhier F, Feron O and Preat V: To exploit the tumor microenvironment: Passive and active tumor targeting of nanocarriers for anti-cancer drug delivery. J Control Release 148: 135-146, 2010.

12. Hanahan D and Weinberg RA: Hallmarks of cancer: The next generation. Cell 144: 646-674, 2011.

13. Carmeliet P and Jain RK: Angiogenesis in cancer and other diseases. Nature 407: 249-257, 2000.

14. Horsman MR, Mortensen LS, Petersen JB, Busk M and Overgaard J: Imaging hypoxia to improve radiotherapy outcome. Nat Rev Clin Oncol 9: 674-687, 2012.

15. Geng L, Donnelly E, McMahon G, Lin PC, Sierra-Rivera E, Oshinka $\mathrm{H}$ and Hallahan DE: Inhibition of vascular endothelial growth factor receptor signaling leads to reversal of tumor resistance to radiotherapy. Cancer Res 61: 2413-2419, 2001.

16. Gottesman MM: Mechanisms of cancer drug resistance. Annu Rev Med 53: 615-627, 2002.

17. Coley WB: The treatment of malignant tumors by repeated inoculations of erysipelas. With a report of ten original cases 1893. Clin Orthop Relat Res: 3-11, 1991.

18. Mose JR and Mose G: Oncolysis by Clostridia. I. Activity of clostridium butyricum (M-55) and other nonpathogenic clostridia against the ehrlich carcinoma. Cancer Res 24: 212-216, 1964.

19. Roberts NJ, Zhang L, Janku F, Collins A, Bai RY, Staedtke V, Rusk AW, Tung D, Miller M, Roix J, et al: Intratumoral injection of clostridium novyi-NT spores induces antitumor responses. Sci Transl Med 6: 249ra111, 2014.
20. Forbes NS: Engineering the perfect (bacterial) cancer therapy. Nat Rev Cancer 10: 785-794, 2010.

21. Wei MQ, Mengesha A, Good D and Anne J: Bacterial targeted tumour therapy-dawn of a new era. Cancer Lett 259: 16-27, 2008.

22. Patyar S, Joshi R, Byrav DSP, Prakash A, Medhi B and Das BK: Bacteria in cancer therapy: A novel experimental strategy. J Biomed Sci 17: 21, 2010.

23. Agrawal N, Bettegowda C, Cheong I, Geschwind JF, Drake CG, Hipkiss EL, Tatsumi M, Dang LH, Diaz LA Jr, Pomper M, et al: Bacteriolytic therapy can generate a potent immune response against experimental tumors. Proc Natl Acad Sci USA 101: 15172-15177, 2004.

24. Bettegowda C, Huang X, Lin J, Cheong I, Kohli M, Szabo SA, Zhang X, Diaz LA Jr, Velculescu VE, Parmigiani G, et al: The genome and transcriptomes of the anti-tumor agent clostridium novyi-NT. Nat Biotechnol 24: 1573-1580, 2006.

25. Staedtke V, Bai RY, Sun W, Huang J, Kibler KK, Tyler BM, Gallia GL, Kinzler K, Vogelstein B, Zhou S and Riggins GJ: Clostridium novyi-NT can cause regression of orthotopically implanted glioblastomas in rats. Oncotarget 6: 5536-5546, 2015.

26. Casares N, Pequignot MO, Tesniere A, Ghiringhelli F, Roux S, Chaput N, Schmitt E, Hamai A, Hervas-Stubbs S, Obeid M, et al: Caspase-dependent immunogenicity of doxorubicin-induced tumor cell death. J Exp Med 202: 1691-1701, 2005.

27. Michaud M, Martins I, Sukkurwala AQ, Adjemian S, Ma Y, Pellegatti P, Shen S, Kepp O, Scoazec M, Mignot G, et al: Autophagy-dependent anticancer immune responses induced by chemotherapeutic agents in mice. Science 334: 1573-1577, 2011.

28. Obeid M, Tesniere A, Ghiringhelli F, Fimia GM, Apetoh L, Perfettini JL, Castedo M, Mignot G, Panaretakis T, Casares N, et al: Calreticulin exposure dictates the immunogenicity of cancer cell death. Nat Med 13: 54-61, 2007.

29. Furumoto K, Soares L, Engleman EG and Merad M: Induction of potent antitumor immunity by in situ targeting of intraturnoral DCs. J Clin Invest 113: 774-783, 2004.

30. DeClue AE, Axiak-Bechtel SM, Zhang Y, Saha S, Zhang L, Tung D and Bryan JN: Immune response to C. novyi-NT immunotherapy. Vet Res 49: 38, 2018.

31. Ji J, Park WR, Cho S, Yang Y, Li W, Harris K, Huang X, Gu S, Kim DH,Zhang Z and Larson AC: Iron-oxide nanocluster labeling of clostridium novyi-NT spores for MR imaging-monitored locoregional delivery to liver tumors in rat and rabbit models. J Vasc Interv Radiol 30: 1106-1115, 2019.

32. Theys J, Landuyt W, Nuyts S, Van Mellaert L, Bosmans E, Rijnders A, Van Den Bogaert W, van Oosterom A, Anné J and Lambin P: Improvement of clostridium tumour targeting vectors evaluated in rat rhabdomyosarcomas. FEMS Immunol Med Microbiol 30: 37-41, 2001.

33. Bettegowda C, Dang LH, Abrams R, Huso DL, Dillehay L, Cheong I, Agrawal N, Borzillary S, McCaffery JM, Watson EL, et al: Overcoming the hypoxic barrier to radiation therapy with anaerobic bacteria. Proc Natl Acad Sci USA 100: 15083-15088, 2003.

34. Krick EL, Sorenmo KU, Rankin SC, Cheong I, Kobrin B, Thornton K, Kinzler KW, Vogelstein B, Zhou S and Diaz LA Jr: Evaluation of clostridium novyi-NT spores in dogs with naturally occurring tumors. Am J Vet Res 73: 112-118, 2012.

35. Hekmatshoar Y, Rahbar Saadat Y, Hosseiniyan Khatibi SM, Ozkan T, Zununi Vahed F, Nariman-Saleh-Fam Z, Pourghassem Gargari B, Sunguroglu A and Zununi Vahed S: The impact of tumor and gut microbiotas on cancer therapy: Beneficial or detrimental? Life Sci 233: 116680, 2019.

36. Umer B, Good D, Anne J, Duan W and Wei MQ: Clostridial spores for cancer therapy: Targeting solid tumour microenvironment. J Toxicol 2012: 862764, 2012.

37. Danino T, Lo J, Prindle A, Hasty J and Bhatia SN: In vivo gene expression dynamics of tumor-targeted bacteria. ACS Synth Biol 1: 465-470, 2012.

38. Li LY, Rojiani A and Siemann DW: Targeting the tumor vasculature with combretastatin A-4 disodium phosphate: Effects on radiation therapy. Int J Radiat Oncol Biol Phys 42: 899-903, 1998.

39. Smith AB III, Freeze BS, LaMarche MJ, Sager J, Kinzler KW and Vogelstein B: Discodermolide analogues as the chemical component of combination bacteriolytic therapy. Bioorg Med Chem Lett 15: 3623-3626, 2005.

40. Dang LH, Bettegowda H, Agrawal N, Cheong I, Huso D, Frost P, Loganzo F, Greenberger L, Barkoczy J, Pettit GR, et al: Targeting vascular and avascular compartments of tumors with C. novyi-NT and anti-microtubule agents. Cancer Biol Ther 3: 326-337, 2004. 
41. Cheong I, Huang X, Bettegowda C, Diaz LA Jr, Kinzler KW, Zhou S and Vogelstein B: A bacterial protein enhances the release and efficacy of liposomal cancer drugs. Science 314: 1308-1311, 2006.

42. Shimose S, Kawaguchi T, Tanaka M, Iwamoto H, Miyazaki K, Moriyama E, Suzuki H, Niizeki T, Shirono T, Nakano M, et al: Lenvatinib prolongs the progression-free survival time of patients with intermediate-stage hepatocellular carcinoma refractory to transarterial chemoembolization: A multicenter cohort study using data mining analysis. Oncol Lett 20: 2257-2265, 2020.

43. Zhang XB: Experimental study of anaerobic bacteria combined with interventional therapy for liver cancer. $\mathrm{PhD}$ dissertation. Fudan University, Shanghai, China, 2009

44. Wachsberger P, Burd R and Dicker AP: Tumor response to ionizing radiation combined with antiangiogenesis or vascular targeting agents: Exploring mechanisms of interaction. Clin Cancer Res 9: 1957-1971, 2003.

45. Garcia-Barros M, Paris F, Cordon-Cardo C, Lyden D, Rafii S, Haimovitz-Friedman A, Fuks Z and Kolesnick R: Tumor response to radiotherapy regulated by endothelial cell apoptosis. Science 300: 1155-1159, 2003.

46. Butterworth KT, Prise KM and Verhaegen F: Small animal image-guided radiotherapy: Status, considerations and potential for translational impact. Br J Radiol 88: 20140634, 2015.

47. Wei MQ, Ellem KA, Dunn P, West MJ, Bai CX and Vogelstein B: Facultative or obligate anaerobic bacteria have the potential for multimodality therapy of solid tumours. Eur J Cancer 43 490-496, 2007.

48. Diaz LA, Cheong I, Foss CA, Zhang X, Peters BA, Agrawal N, Bettegowda C, Karim B, Liu G, Khan K, et al: Pharmacologic and toxicologic evaluation of C-novyi-NT spores. Toxicol Sci 88: $562-575,2005$.

49. Kaimala S, Al-Sbiei A, Cabral-Marques O Fernandez-Cabezudo MJ and Al-Ramadi BK: Attenuated bacteria as immunotherapeutic tools for cancer treatment. Front Oncol 8: 36, 2018.

50. Bos R, Zhong H, Hanrahan CF, Mommers EC, Semenza GL, Pinedo HM, Abeloff MD, Simons JW, van Diest PJ and van der Wall E: Levels of hypoxia-inducible factor-1 alpha during breast carcinogenesis. J Natl Cancer Inst 93: 309-314, 2001.

51. Groot AJ, Mengesha A, van der Wall E, van Diest PJ, Theys J and Vooijs M: Functional antibodies produced by oncolytic clostridia. Biochem Biophys Res Commun 364: 985-989, 2007.
52. Abdalla EK, Vauthey JN, Ellis LM, Ellis V, Pollock R, Broglio KR, Hess K and Curley SA: Recurrence and outcomes following hepatic resection, radiofrequency ablation, and combined resection/ablation for colorectal liver metastases. Ann Surg 239: 818-824, 2004.

53. Dietzel F, Gericke D and Konig W: Tumor hyperthermia using high frequency for increase of oncolysis by clostridium butyricum (M 55). Strahlentherapie 152: 537-541, 1976 (In German).

54. Dietzel F and Gericke D: Intensification of the oncolysis by clostridia by means of radio-frequency hyperthermy in experiments on animals-dependence on dosage and on intervals (author's transl). Strahlentherapie 153: 263-266, 1977 (In German).

55. Gericke D, Dietzel F, Konig W, Ruster I and Schumacher L: Further progress with oncolysis due to apathogenic clostridia. Zentralbl Bakteriol Orig A 243: 102-112, 1979.

56. Lee $\mathrm{CH}$ : Engineering bacteria toward tumor targeting for cancer treatment: Current state and perspectives. Appl Microbiol Biotechnol 93: 517-523, 2012.

57. Thiele EH, Arison RN and Boxer GE: Oncolysis by clostridia. IV. Effect of nonpathogenic clostridial spores in normal and pathological tissues. Cancer Res 24: 234-238, 1964.

58. Liu G, Bettegowda C, Qiao Y, Staedtke V, Chan KWY, Bai R, Li Y, Riggins GJ, Kinzler KW, Bulte JWM, et al: Noninvasive imaging of infection after treatment with tumor-homing bacteria using chemical exchange saturation transfer (CEST) MRI. Magn Reson Med 70: 1690-1698, 2013.

59. Park W, Cho S, Huang X, Larson AC and Kim DH: Branched gold nanoparticle coating ofclostridium novyi-NT spores for CT-guided intratumoral injection. Small 13: 10, 2017.

60. Zheng L, Zhang Z, Khazaie K, Saha S, Lewandowski RJ, Zhang $\mathrm{G}$ and Larson AC: MRI-monitored intra-tumoral injection of iron-oxide labeled clostridium novyi-NT anaerobes in pancreatic carcinoma mouse model. PLos One 9: 116204, 2014.

This work is licensed under a Creative Commons Attribution-NonCommercial-NoDerivatives 4.0 International (CC BY-NC-ND 4.0) License. 